PERSPECTIVE

\title{
The management of retinal vein occlusion: is interventional ophthalmology the way forward?
}

\author{
H Shahid, P Hossain, W M Amoaku
}

Br J Ophthalmol 2006;90:627-639. doi: 10.1136/bjo.2005.068668

Retinal vein occlusions (RVO) are the second commonest sight threatening vascular disorder. Despite its frequency treatments for RVO are unsatisfactory and include several that have not been tested by large, well designed, prospective, randomised controlled trials. There is also the lack of long term follow up in many of the available small uncontrolled studies, and the timings of interventions are haphazard. This review aims to evaluate the current knowledge relating to the pathogenesis, suggested treatments for the different types of RVO, and their complications. Isovolaemic haemodilution is of limited benefit and should be avoided in patients with concurrent cardiovascular, renal, or pulmonary morbidity. Evidence to date does not support any therapeutic benefit from radial optic neurotomy, optic nerve decompression, or arteriovenous crossing sheathotomy on its own. Vitrectomy combined with intravenous thrombolysis may offer promise for central RVO. Similarly, vitrectomy combined with arteriovenous sheathotomy intravenous tissue plasminogen activator may offer benefits for branch RVO. RVOs occur at significantly high frequency to allow future prospective randomised controlled studies to be conducted to evaluate the role of different therapeutic modalities singly or in combination.

See end of article for authors' affiliations

Correspondence to: Mr W M Amoaku, Academic Department of Ophthalmology, University of Nottingham, Eye, Ear, Nose and Throat Centre, Queen's Medical Centre, Nottingham NG7 2UH,

UK; wma@nottingham.ac. uk

Accepted for publication 9 December 2005
$\mathrm{R}$ etinal vein occlusion (RVO) is the second most common retinal vascular disorder after diabetic retinopathy and is a significant cause of visual handicap. ${ }^{1-4}$ The prevalence of retinal vein occlusions has been shown to vary from $0.7 \%$ to $1.6 \% .^{5}$ In the United Kingdom retinal vein occlusions are responsible for a significant number of new blind registrations per annum and eye enucleations subsequent to neovascular complications.

There are two main types of retinal vein occlusion-central retinal vein occlusion (CRVO) and branch retinal vein occlusion (BRVO). Our knowledge of the outcome of patients with CRVO and BRVO has been enhanced by the findings of the Central Vein Occlusion Study (CVOS) ${ }^{3}$ and the Branch Vein Occlusion Study groups (BVOS). ${ }^{16}$

According to the CVOS, the final visual acuity after CRVO depends on the visual acuity at presentation. Vision did not improve in $80 \%$ patients with vision less than $6 / 60(20 / 200)$ at presentation but $65 \%$ of patients with vision better than 6/12 (20/40) maintained this level of acuity at the end of follow up. ${ }^{3}$

The visual prognosis following CRVO is largely related to the residual level of venous perfusion. In ischaemic (non-perfused) CRVO the final visual acuity is $3 / 60(20 / 400)$ or worse in $87 \%$ of cases, whereas for non-ischaemic cases the vision is $6 / 9(20 / 30)$ or better in $57 \%{ }^{7}$ Additionally, the subsequent development of macular oedema and neovascularisation has adverse effects on vision. Panretinal laser photocoagulation helps to reduce the progress of neovascular complications. However, the use of grid macular laser photocoagulation to reduce macular oedema has not been found to improve visual acuity in patients with persistent macular oedema. $^{368}$

BRVO is more common than CRVO and has a more favourable prognosis. The BVOS reported that, overall, $50-60 \%$ patients with BRVO will maintain visual acuity of 6/12 (20/40) or better after 1 year and thereafter the natural course of BRVO remains relatively static. ${ }^{2}$

Visual loss following BRVO may result from macular oedema, foveal haemorrhage, vitreous haemorrhage, epiretinal membrane, retinal detachment, macular ischaemia, and neovascular complications. The BVOS demonstrated that the probability for retinal neovascularisation is significantly greater in eyes with an area of retinal non-perfusion that measures 5 disc diameters or more and that the use of laser photocoagulation is beneficial for patients who develop macular oedema or retinal neovascularisation and have visual acuities of $20 / 40$ to $20 / 100{ }^{9}$

Until recently, the management of vein occlusions has been largely dependent on the results of the CVOS and BVOS. Patients are investigated to exclude any underlying systemic or ocular risk factors and treatment is given for sight threatening sequelae. However, over the past decade more interventional therapeutic options have emerged. The new treatments are targeted at the presumed underlying mechanical and physiological factors that lead to venous occlusion (fig 1).

Abbreviations: $A-V$, arteriovenous; BRVO, branch retinal vein occlusion; BVOS, Branch Vein Occlusion Study; CRVO, central retinal vein occlusion; CVOS, Central Vein Occlusion Study; ETDRS, Early Treatment Diabetic Retinopathy Study; MVR, microvitreoretinal; PRP, panretinal photocoagulation; RVO, retinal vein occlusion; t-PA, tissue plasminogen activator; VEGF, vascular endothelial growth factor 


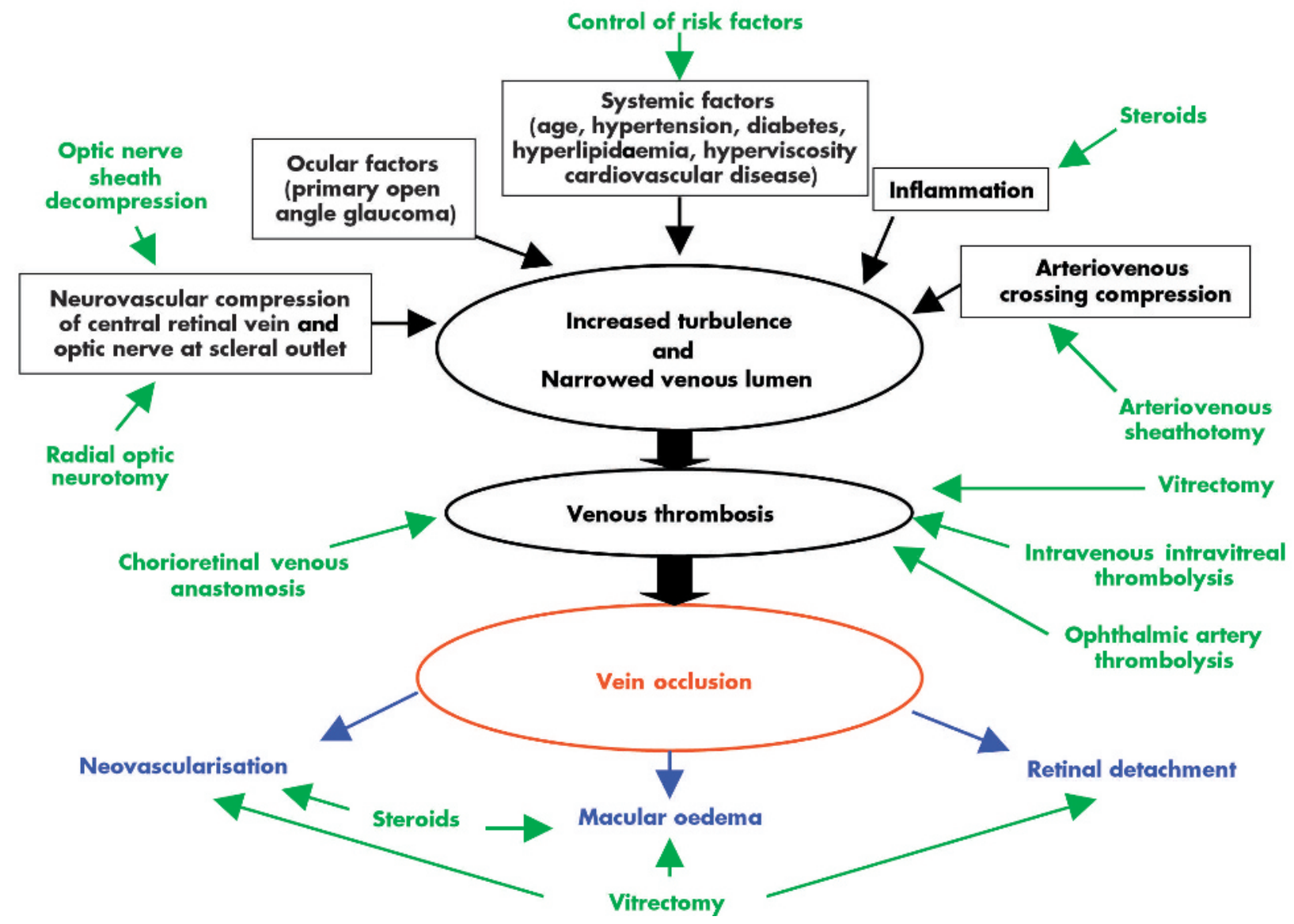

Figure 1 Outline of current treatments for RVO. Treatment modalities indicated in green, possible aetiological factors and mechanisms indicated in black/red, and complications of vein occlusion indicated in blue.

This review aims to put into perspective the emerging treatment options for both CRVO and BRVO and discuss whether they offer a beneficial alternative over the natural course of the disease (tables 1 and 2). A summary of the main studies described in the literature is given in tables 3 and 4 .

\section{INTERVENTIONS FOR CRVO}

\section{Steroids}

The use of steroids is advocated by those who propose an inflammatory mechanism for the pathogenesis of CRVO. Systemic steroid treatment may be considered in patients with non-ischaemic CRVO with an inflammatory component. ${ }^{10}$ This may be particularly justified in patients with an

\begin{tabular}{l} 
Table 1 Summary of interventional methods \\
for central retinal vein occlusion (CRVO) \\
\hline Interventions for CRVO \\
\hline Steroids \\
Thrombolysis \\
intravitreal thrombolysis \\
retinal venous thrombolysis \\
Isovolaemic haemodilution \\
Radial optic neurotomy \\
Chorioretinal anastamosis \\
Optic nerve sheath decompression \\
Lamina puncture (experimental) \\
\hline
\end{tabular}

underlying systemic vasculitic disorder or younger patients who classically tend to have papillophlebitis. In a report of two patients pulse steroid therapy was found to increase visual acuity from $3 / 60(20 / 800)$ to $6 / 7.5(20 / 25)$ and from $6 / 24(20 / 80)$ to $6 / 12(20 / 40) .^{11}$

Local injection of steroid into the vitreous limits the side effects of systemic steroid therapy and is shown to give both anatomical (as shown by optical coherence tomography) and functional (visual acuity) improvement in macular oedema following vein occlusion. ${ }^{12-20}$ Steroids may work by stabilising the blood-retina barrier. ${ }^{21}$ In vitro corticosteroids inhibit vascular endothelial growth factor (VEGF) expression ${ }^{22}$ and may thus prevent the neovascular sequelae of vein occlusion and reduce VEGF mediated retinal capillary permeability. ${ }^{18}$

Intravitreal steroids, particularly triamcinolone, seem effective in treating neovascularisation and macular oedema for both ischaemic and non-ischaemic types of vein

Table 2 Summary of interventional methods for branch retinal vein occlusion (BRVO)

Interventions for BRVO

Steroids

Isovolaemic haemodilution

Vitrectomy

Arteriovenous crossing sheathotomy

Chorioretinal anastomosis 


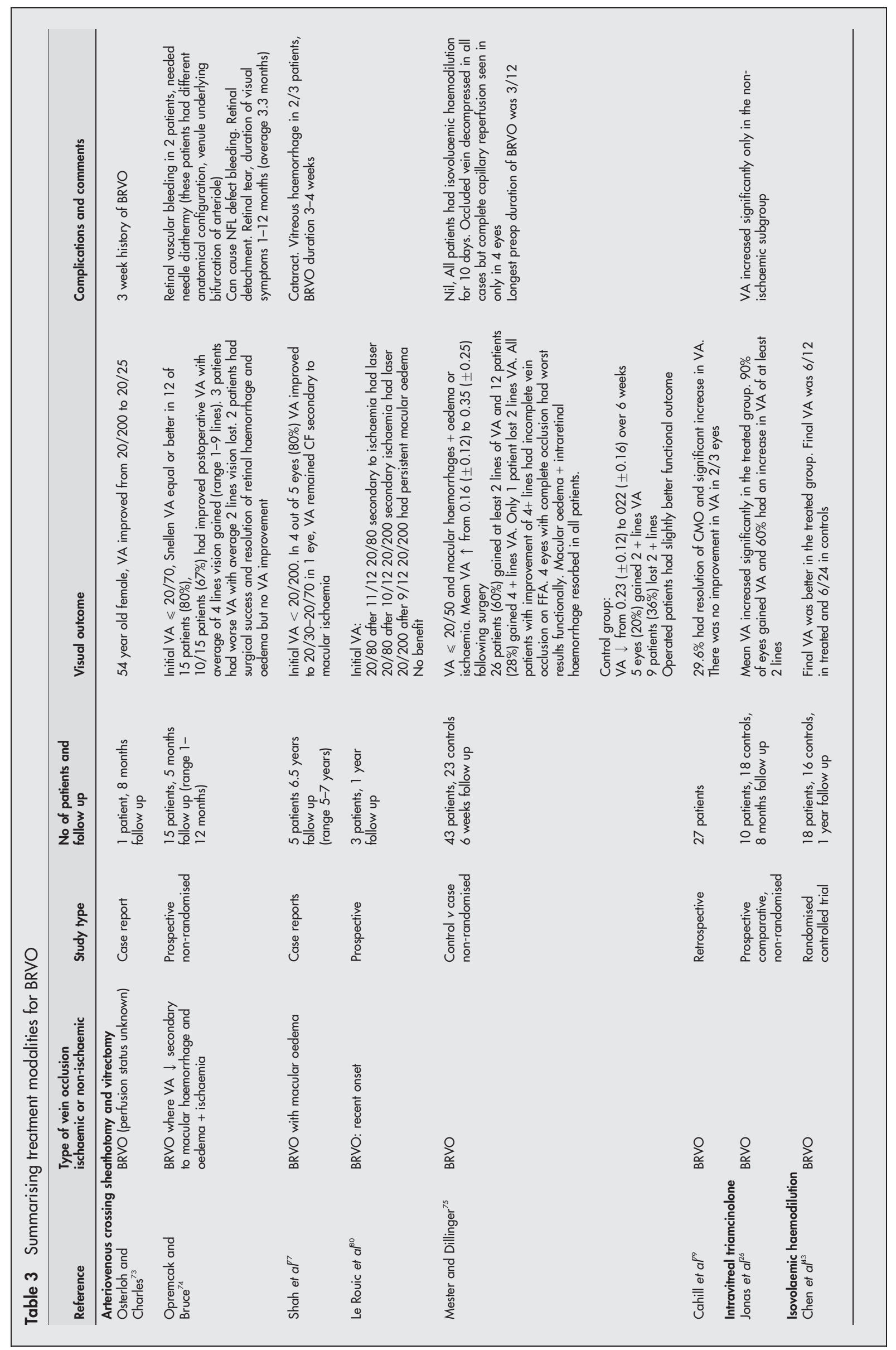




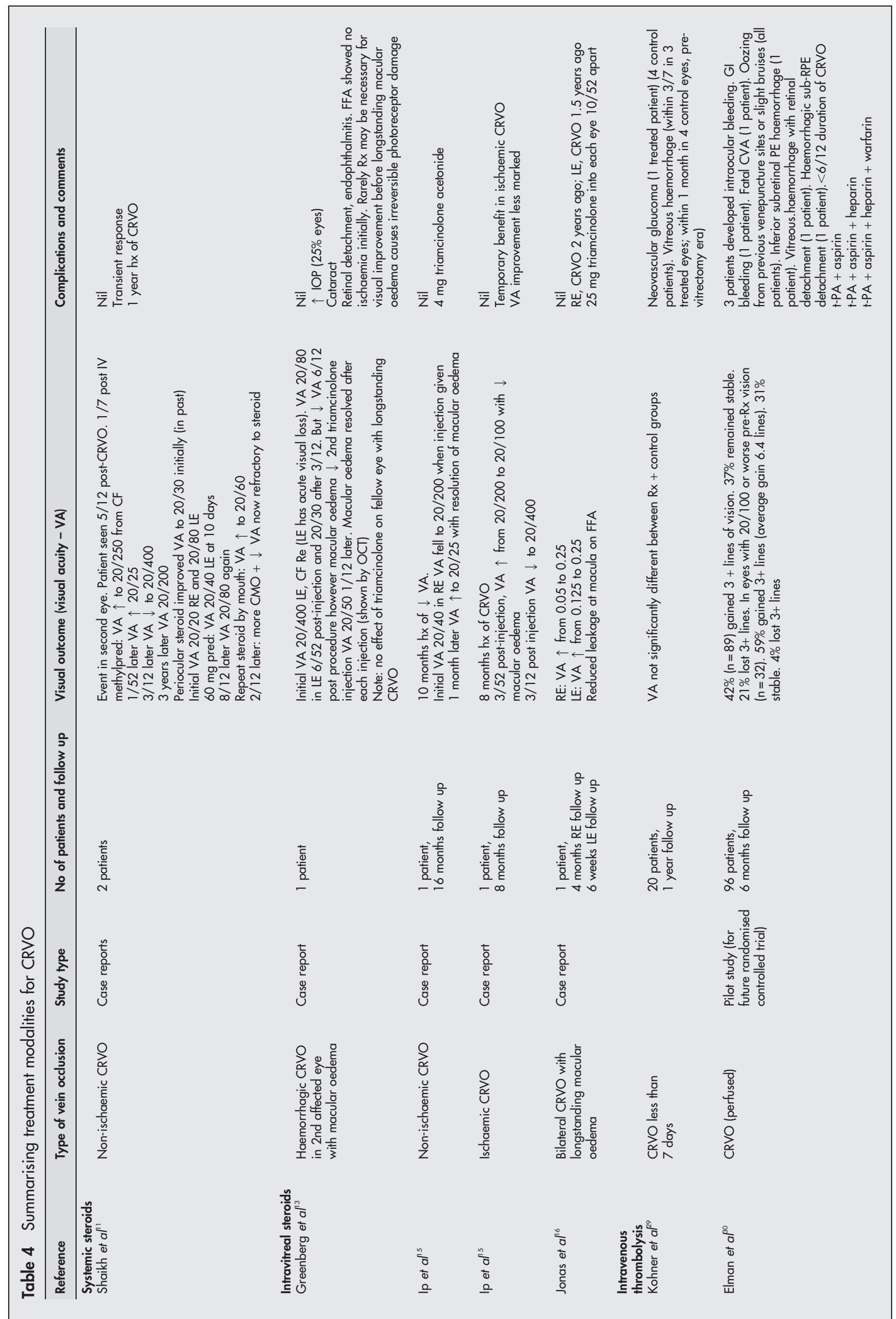




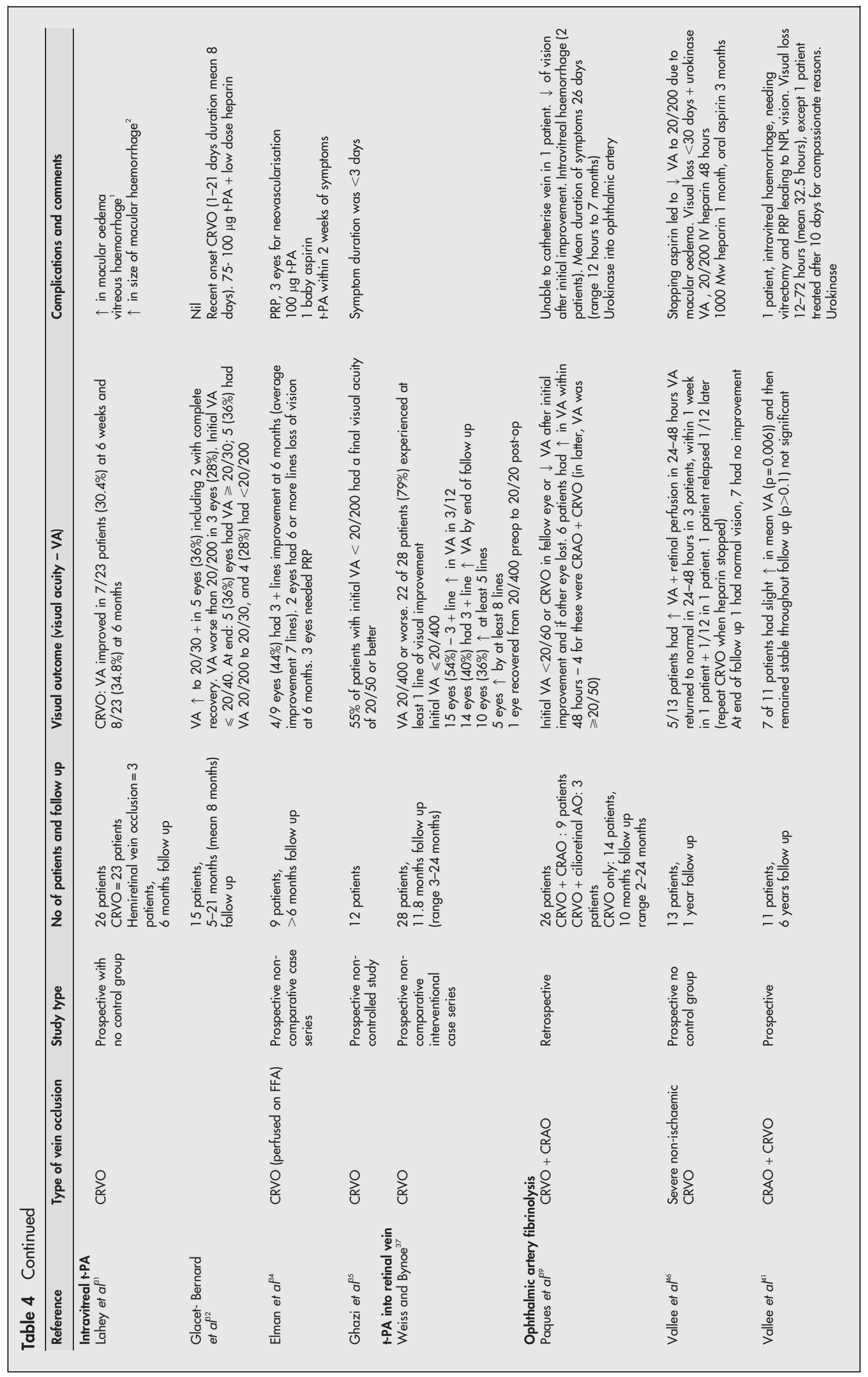



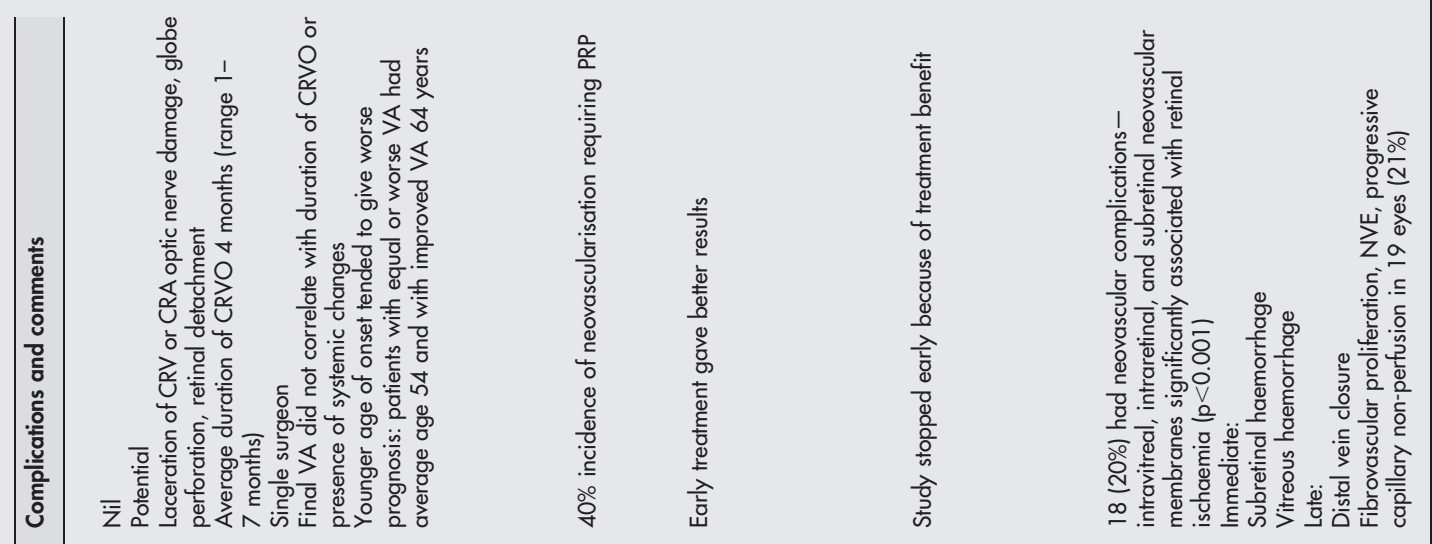

वำ

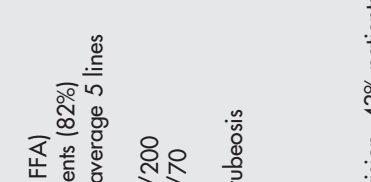

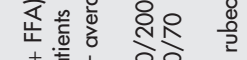

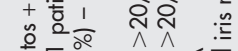

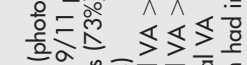

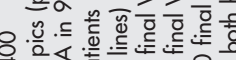

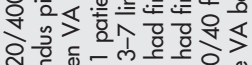
Vं

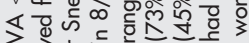

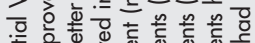

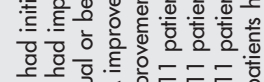

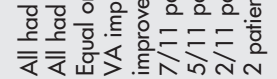

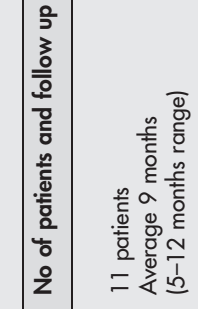



:

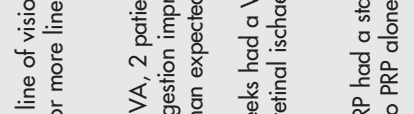

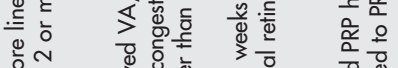



के

.

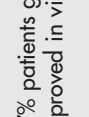

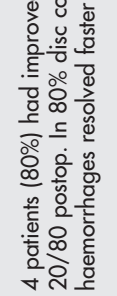

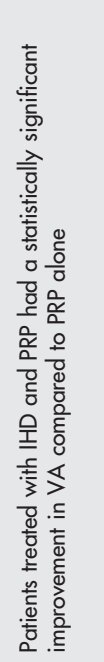

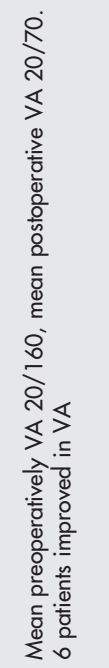

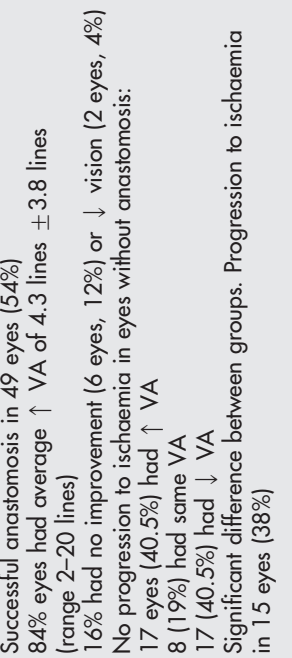

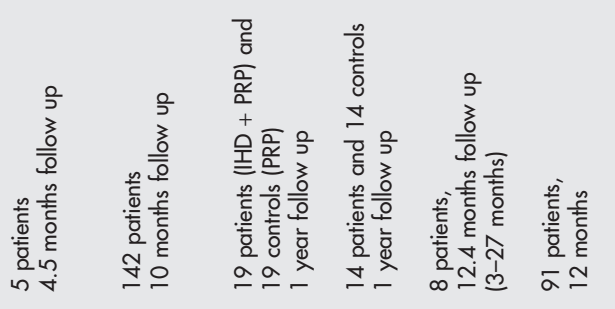


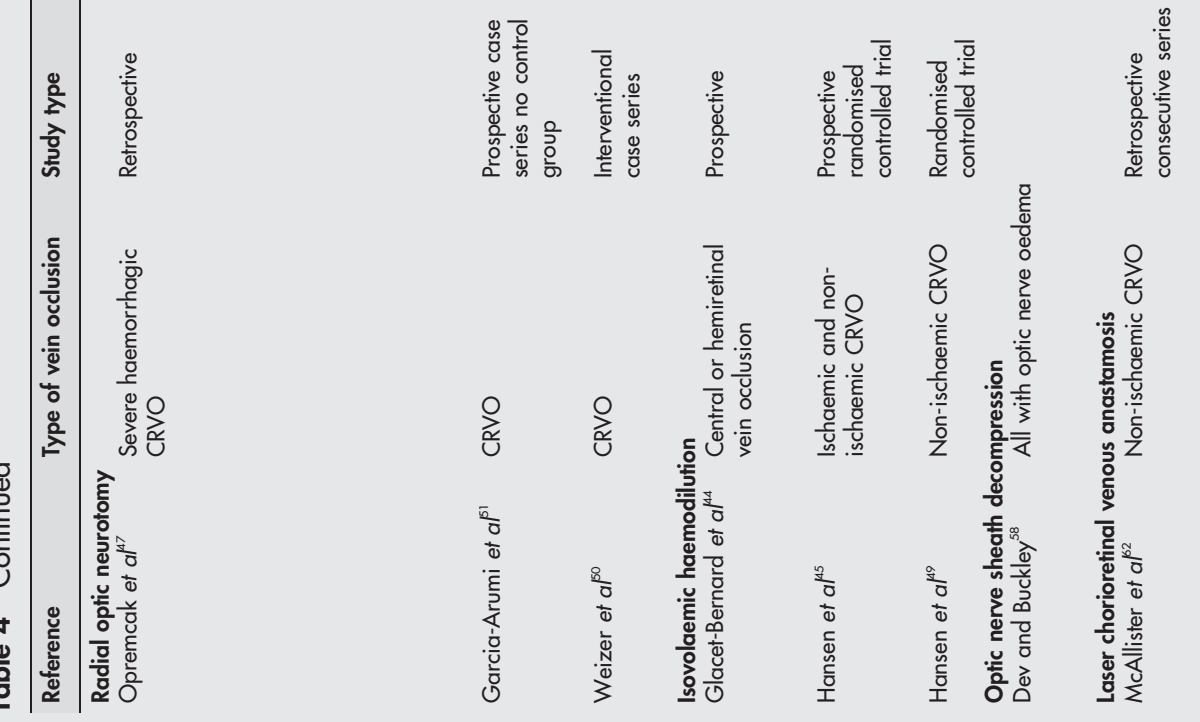


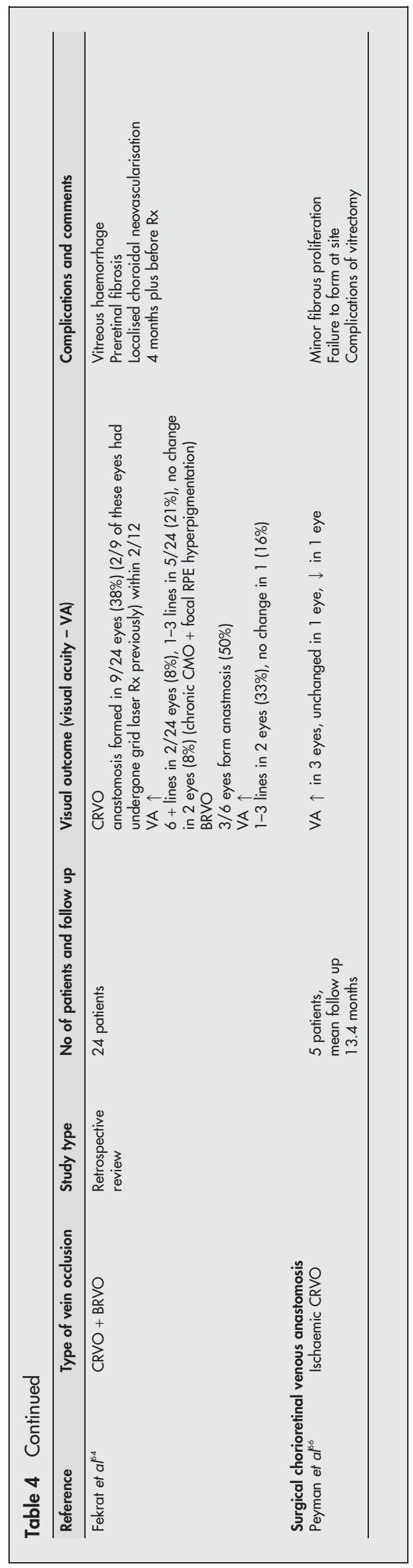

occlusion. ${ }^{16} 23-25$ Intravitreal steroid injection is a quick procedure that can be performed in an outpatient setting. Steroids are an option when patients with macular oedema are not eligible for or are resistant to laser photocoagulation. However, since the response appears short lived ${ }^{11}{ }^{13}{ }^{15}$ steroids may be not be suitable as definitive treatment. The risks of steroid administration must also be considered. Raised intraocular pressure is reported to occur in up to $25 \%$ eyes after intravitreal steroid ${ }^{20}$ and can make the eye prone to further ischaemic insult. The development of intractable glaucoma has also been reported. ${ }^{26}$ Other risks include infection, cataract, haemorrhage, and retinal detachment.

In chronic macular oedema there is a suggestion that anatomical success does not equate with functional improvement. ${ }^{13}$ Perhaps early treatment is necessary for visual improvement before longstanding macular oedema results in irreversible photoreceptor damage. Until a large casecontrol or randomised controlled series is available recommendations cannot be made on dosage, timing, and case selection for the use of steroids.

\section{Anticoagulation and thrombolysis}

It is postulated that dissolving the thrombus causing CRVO at the lamina cribrosa and/or preventing its reformation (through anticoagulation or antiplatelet therapy) should effectively restore retinal blood flow and visual function. Even if irreversible damage has occurred rapid restoration of normal retinal blood flow, through clot dissolution, may prevent further visual loss from the chronic complications of CRVO.

Systemic anticoagulants (such as oral aspirin, subcutaneous heparin, or intravenous thrombolysis) have not been shown to be an effective treatment for vein occlusions. ${ }^{27}{ }^{28} \mathrm{In}$ addition, their use causes unacceptable risks of intraocular, intracerebral, and gastrointestinal haemorrhage. ${ }^{29}{ }^{30}$ Consequently, local routes of administration of thrombolytic agents have been investigated.

\section{Intravitreal thrombolysis}

The rationale for intravitreal administration of tissue plasminogen activator (t-PA) is that it can diffuse across the internal limiting membrane of the retina and enter the retinal circulation through capillaries damaged by breakdown of the blood-retina barrier following CRVO. The t-PA would then be transported by residual venous flow towards the lamina cribrosa, the presumed site of thrombus, to cause thrombus lysis.

Lahey et al reported that eight (34.8\%) of 23 patients achieved vision greater than or equal to 6/12 (20/40) at 3 months after intravitreal injection of t-PA. This included an eye that went from $3 / 60(20 / 400)$ to $6 / 7.5(20 / 25)$ and another from $1 / 60(3 / 200)$ to $6 / 6(20 / 20) .{ }^{31}$ In the CVOS, only $3.2 \%$ of 155 eyes achieved this level of vision at 4 months. GlacetBernard et al also found that vision improved to at least 20/30 in $36 \%$ eyes following intravitreal thrombolysis. ${ }^{32}$ Success was not universal. This may reflect the varying maturity of the clots at the time of intervention, as animal studies suggest that t-PA is most effective in immature clots. ${ }^{33}$ Co-existent poor retinal perfusion may limit visual improvement. Important factors that seemed to determine outcome were the type of CRVO, and the timing of intervention. However, the lack of a control group in these studies and the small patient numbers means there is insufficient material for substantive conclusions to be reached.

Complications of intravitreal thrombolysis include vitreous haemorrhage and an increase in macular oedema. ${ }^{31}{ }^{32}$ It is suggested that t-PA may have lysed smaller secondary fibrin clots at the retinal capillary level that are deposited in response to the damage caused by the vein occlusion. If this occurs without the clot at the lamina cribrosa being lysed, the 
retinal venous pressure remains elevated and there may be an increase in exudation which can lead to increased macular oedema. The most dramatic improvement following t-PA was seen in non-ischaemic CRVO. ${ }^{34} 35$

\section{Retinal venous thrombolysis}

Retinal vein cannulation allows t-PA to be delivered under direct view into the retinal vein to cause thrombolysis. After the initial report of this procedure ${ }^{36}$ Weiss and Bynoe conducted a prospective interventional case series of 28 patients with an average duration of CRVO of 4.9 months and visual acuity of $3 / 60(20 / 400)$ or worse ${ }^{37} ; 22$ of the 28 patients $(79 \%)$ who underwent the procedure had better visual acuity at the final follow up examination than at baseline. Ten eyes (36\%) improved by at least five lines of vision at the last follow up and five eyes improved by at least eight lines. One eye recovered from 3/60 (20/400) preoperatively to $6 / 6(20 / 20)$ postoperatively. Side effects were, however, significant. Vitreous haemorrhage was seen in seven patients $(25 \%)$ and one patient went on to develop a retinal detachment.

Weiss and Bynoe propose that direct venous thrombolysis provides a high local concentration of t-PA which is delivered at a high flow rate and can act to flush out the thrombus. However, the law of haemodynamics suggest that when CRVO is longstanding the t-PA would follow the path of least resistance and be flushed out of the central retinal vein via the collateral vessels that have developed with little t-PA remaining to act on the thrombus. ${ }^{38}$ However, if t-PA is injected early after onset of the vein occlusion, collateral vessels will not yet have developed and the t-PA may then reach the desired site. Further studies are needed on the optimal timing for intervention.

\section{Ophthalmic artery thrombolysis}

Recent advances in interventional neuroradiology have made it possible to selectively catheterise the ophthalmic artery and infuse a fibrinolytic agent close to the obstruction site.

Three studies have been reported using this novel technique in patients with CRVO. ${ }^{39-41}$ The internal carotid artery was catheterised via the femoral artery. An initial arteriogram was used to fashion the tip of the microcatheter in accordance with the geometry of the carotid arterial siphon and ophthalmic arterial ostium. Urokinase was then infused over 1 hour. Subsequently, all patients were given intravenous heparin for 48 hours, followed by subcutaneous low molecular weight heparin for 1 month (to inhibit coagulation on the thrombus and limit clot extension and recurrence) and oral aspirin for 3 months (to combat risk factors for atherosclerosis and prevent further thrombosis).

In a retrospective study of 26 patients (all with VA less than 6/18 (20/60)) six patients had improved vision after 48 hours, which was maintained long term in five patients. ${ }^{39}$ In a subgroup analysis, four of the six patients with visual improvement had pretreatment Funduscopic appearances suggestive of combined central retinal artery and vein occlusion. This pattern was seen in nine of 26 patients in the series. Thus, overall, four out of the nine $(44 \%)$ patients with this pattern improved.

In a prospective series of five patients, three who had the features of combined central retinal artery and vein occlusion achieved full visual recovery after this intervention. ${ }^{40}$ These patients had visual loss of less than 30 days' duration with visual acuity less than $6 / 30(20 / 100)$. These patients had no capillary closure, and four of them had no macular oedema. It seems, therefore, that impairment of retinal arterial perfusion was responsible for their visual loss and minimally invasive local intra-arterial thrombolysis had a rapid beneficial effect.
Since patients with combined central retinal artery and vein occlusion were found to respond well to thromboly$\operatorname{sis}^{39}{ }^{40}$ a separate prospective study was undertaken on this group of patients. ${ }^{41}$ Eleven consecutive patients with visual loss of less than 72 hours were treated during a 6 year period. Vision improved at some point after treatment in seven of 11 patients, including six in who substantial improvement after 24-48 hours. Funduscopy showed the retinal pathology to regress over $2-4$ weeks.

Complications encountered include vitreous haemorrhage which may lead to blindness. ${ }^{41}$ The haemorrhage may have resulted from rupture of ischaemic retinal vessels caused by severely impaired retinal arterial perfusion as well as a combined sudden increase in retinal venous hydrostatic pressure caused by venous thrombosis as a result of the release of arterial thrombosis by fibrinolysis. Local administration of thrombolytics avoids systemic complications or change in clotting status. There were no cases of postprocedure neurological deficit.

There is the possible requirement for long term anticoagulation following thrombolysis. One patient had a repeat CRVO just over 1 month after thrombolysis when heparin was stopped. Similarly, stopping aspirin led to a further decrease in vision from $6 / 15(20 / 50)$ to $6 / 60(20 / 200)$ as a result of cystoid macular oedema. ${ }^{40}$

The local administration of thrombolytic agents is a feasible treatment for RVO but may only be beneficial in certain types of RVOs. In addition, it seems appropriate to administer treatment before the thrombus becomes organised. Promising results are seen in patients with combined central retinal artery and vein occlusion. Unfortunately, the studies lack a control group and spontaneous improvement of this subgroup has been reported. ${ }^{42}$

\section{Isovolaemic haemodilution}

Whatever the primary cause of RVO, the obstruction causes a local increase in blood viscosity. In isovolaemic haemodilution, venesection is performed at the same time as infusion of a plasma substitute (for example, hydroxyethyl starch, dextran) so that a constant blood volume is maintained. By reducing the haematocrit level, haemodilution causes a reduction in blood viscosity and thus improves retinal blood flow and oxygen supply. Thus, it relieves retinal hypoperfusion during a critical time of occlusion and may prevent capillary closure and further retinal ischaemia.

Haemodilution therapy has been shown to be an effective intervention for CRVO in a number of randomised studies. Beneficial effects have been seen with respect to visual acuity, retinal circulation times, and haemorheological parameters. ${ }^{43-46}$

Hansen et al carried out a prospective randomised trial evaluating the efficacy of combined isovolaemic haemodilution and panretinal photocoagulation (PRP) in patients with ischaemic and non-ischaemic CRVO. Patients treated with haemodilution and PRP showed a statistically significant improvement in vision compared to patients treated with PRP alone up to 1 year later. In both groups the final visual acuity was worse for ischaemic CRVO. However, the most marked improvement in visual acuity was seen in patients with ischaemic CRVO but this did not reach statistical significance as the numbers of patients were small. ${ }^{45}$

Hansen et al demonstrated a significant treatment effect with the use of isovolaemic haemodilution alone in nonischaemic CRVO. Eight of 14 patients with non-ischaemic CRVO who were given isovolaemic haemodilution alone had improved in visual acuity compared to none of the 11 controls $(\mathrm{p}<0.01){ }^{46}$

The importance of early intervention was shown by GlacetBernard et al, who analysed 142 patients with central or 
hemiretinal vein occlusion. Final visual acuity was significantly better if treatment was instituted within the first 2 weeks of vein occlusion; $41 \%$ had a final visual acuity of $20 / 40$ or better in this early treatment group versus $23 \%$ in the late treatment group $(\mathrm{p}<0.01) .{ }^{44}$

Ischaemic change may underlie the need for early intervention. In Glacet-Bernard's study retinal ischaemia was not significantly greater in the late treatment group at inclusion but at the end of follow up ischaemic change was significantly greater in the late treatment group. Isovolaemic haemodilution thus seems to prevent retinal ischaemia and risk of conversion of non-ischaemic RVO to ischaemic RVO.

Isovolaemic haemodilution is a systemic treatment. Patients having this treatment would need to be carefully selected. It may not be suitable for patients with CCF, renal or respiratory insufficiency and anaemia. Isovolaemic haemodilution alone has not been shown to prevent neovascular glaucoma or to arrest ischaemia and therefore most studies have used adjunctive laser therapy. ${ }^{44}{ }^{45}$

\section{Radial optic neurotomy}

It is thought that radial optic neurotomy releases pressure in the scleral outlet compartment (the space containing the scleral canal, cribriform plate, optic nerve, central retinal artery and vein). This increases the central retinal vein lumen which in turn increases venous flow and helps clear the thrombus.

Examination of cadaveric eyes showed that the scleral outlet could be decompressed via an internal vitreoretinal approach. ${ }^{47}$ A standard three port vitrectomy is done and the intraocular pressure raised to minimise any potential bleeding. A radial optic neurotomy is done using a microvitreoretinal (MVR) blade on the nasal side of the disc to avoid injury to the papillomacular bundle. The cut is radial (nonradial cuts would transect the nerve fibre layer neurons as they enter the optic nerve) and approaches the centre of the cribriform plate.

The Radial Optic Neurotomy Study is a retrospective nonrandomised pilot study of 11 consecutive patients with severe haemorrhagic CRVO. ${ }^{47}$ In the selected patient group, with visual acuity equal to or less than $3 / 60(20 / 400)$, prognosis is normally poor without intervention. ${ }^{3}$ Surgical relaxation of the scleral outlet was associated with a dramatic and rapid clearing of intraretinal haemorrhages and improved retinal blood flow in all patients. Visual acuity improved in eight of the 11 patients $(73 \%)$ with an average of five Snellen lines improvement. The final visual acuity did not correlate with duration of CRVO or the presence of systemic disease. A younger age of onset tended to show a poorer visual prognosis.

These impressive results have been criticised by some authors, who suggest that the use of Snellen acuity (rather than the Early Treatment Diabetic Retinopathy Study (ETDRS) acuity used in the CVOS) may have underestimated the actual presenting visual acuity, and that these patients may have had favourable natural outcomes without intervention. ${ }^{48}$ Retinal capillary non-perfusion was used as the only criterion to make the distinction between ischaemic and non-ischaemic CRVO. Unfortunately, this criterion on its own has poor reliability in making such differentiation in the early stages of CRVO. ${ }^{49}$ However, the distinction is vital in determining prognosis. Further, no visual field evaluations were undertaken in this study and as such no comment could be made on the occurrence of optic nerve damage.

More modest results are seen in a small interventional case series of five patients, ${ }^{50}$ where a $40 \%$ incidence of rubeosis iridis was reported post-procedure. Similarly, modest results are reported by Garcia-Arumi et al, where $43 \%$ patients improved in visual acuity by two or more lines in an uncontrolled study. ${ }^{51}$

Potential complications include laceration of the central retinal artery or nerve, optic nerve damage, globe perforation, and retinal detachment. Visual field defects may occur because of optic nerve head ischaemia after cutting the circle of Zinn-Haller or nerve fibre transection. ${ }^{52}$ Currently, the anatomical success of the technique outweighs functional success and prospective randomised studies are needed.

The rationale for this procedure is difficult to understand. The presence of a hypothetical bottleneck at the optic nerve head is not logical given that the difference in diameter between retrolaminar and prelaminar optic nerves could be explained by the presence of a myelin sheath. The theory would also not be able to explain the development of a hemiretinal vein occlusion..$^{53}$ The central retinal vein lies temporal to the artery in the optic nerve, enclosed by a common fibrous capsule. Thus, the nasal cut in radial optic neurotomy cannot decompress the vein. ${ }^{54}$

\section{Optic nerve sheath decompression}

The rationale for optic nerve sheath decompression is that optic nerve oedema results in some degree of mechanical venous compression. This hypothesis is based on previous studies on posterior scleral ring section ${ }^{55}$ and the demonstration of decreased retinal vein calibre after optic nerve sheath decompression in patients with pseudotumour cerebri. $^{57}$

Optic nerve sheath decompression was performed in eight patients with progressive CRVO and optic nerve oedema. The mean follow up period was 12.4 months. ${ }^{58}$ Overall, six patients improved in visual acuity. Mean preoperative vision was $6 / 48(20 / 160)$ and mean postoperative vision was $6 / 21$ (20/70). The probability of improvement in visual acuity is reported to be $75 \%$. Non-ischaemic eyes operated on within 3 months of CRVO onset, age under 65 years, and initial visual acuity greater than $6 / 60(20 / 200)$ appear to have had better outcomes.

These data seem to argue for a potential role of this procedure in selected cases. However, without a randomised controlled trial and a larger number of patients it is difficult to assess the efficacy of the procedure for this indication.

\section{Lamina puncture}

This is transvitreal optic disc surgery to create a perivascular opening in the lamina cribrosa. This aims to release the postulated constriction of the central retinal vein by the surrounding connective tissue at the level of the lamina cribrosa. The resultant increase in luminal diameter of the central retinal vein may allow the passage of the thrombus or relieve the pressure predisposing to thrombus formation. Alternatively, it may allow sufficient blood flow at the level of the lamina cribrosa to permit increased retinal perfusion, even if the thrombus is not dislodged or mechanically expressed. Thus far the technical feasibility has been established only on human cadaver and enucleated pig eyes. ${ }^{59}$ Until in vivo trials are performed it is not possible to determine if this will lead to success.

\section{Chorioretinal venous anastomosis}

First described almost 50 years ago by Verhoeff, ${ }^{60}$ chorioretinal anastomosis allows blood to bypass the occluded vein into the choroidal circulation. It is thought that vision is improved by a reduction in macular oedema and in progression to an ischaemic status.

This technique has been applied to both BRVO and CRVO and may be done either by laser or surgery. Patient numbers for BRVO are too low for meaningful evaluation. 


\section{Chorioretinal venous anastomosis by laser}

The effect of laser induced chorioretinal anastomosis depends on the type of RVO and the establishment of a successful anastomosis. The best results of this procedure have been found in non-ischaemic CRVO where approximately one third to one half of patients develop a functionally effective chorioretinal anastomosis. Chorioretinal venous anastomosis in ischaemic eyes is very difficult to achieve, perhaps because of the presence of endothelial cell damage. ${ }^{61}$ Although a successful anastomosis prevents further progression towards ischaemia, ${ }^{62}$ the visual results of chorioretinal anastomosis are poor. ${ }^{62-65}$

Complications of this procedure include vitreous haemorrhage $(42 \%)$, choroidal neovascularisation $(21 \%)$, and preretinal fibrosis (12\%). ${ }^{62}$ Complications are magnified in ischaemic CRVO. ${ }^{62}$

In order to reduce the risk of neovascular complications, chorioretinal venous anastomosis should perhaps be limited to non-ischaemic CRVO and patients treated by this technique should be closely followed up. Optimal timing of intervention is unclear but one study suggests it should be less than 4 months, since the average time for the anastomosis to become functional is 6.7 weeks and because an average of 2.1 attempts are needed to establish an anastomosis. $^{62}$

Much work is needed in this field. The ophthalmoscopic and angiographic criteria required to determine the presence of chorioretinal anastomosis have not yet been well described. Further evaluation of the laser power required to produce an anastomosis is necessary. Reasons why some eyes respond to laser by forming anastomosis while others develop a fibrovascular response are unclear. Factors that determine whether or not an anastomosis forms include patient's age, presence of optic nerve head collaterals, the presence of an underlying choroidal vein near the treatment site, and degree of intravascular pressure elevation in the obstructed vein.

\section{Chorioretinal venous anastomosis by surgery}

Given the unpredictable yield of laser induced chorioretinal anastomosis, surgical methods are being evaluated. In a pilot study pars plana vitrectomy was followed by slit-like incisions through Bruch's membrane adjacent to a major branch of a retinal vein in each quadrant. ${ }^{66}$ Small pieces of 5/0 madrilene suture were placed over the vein and inserted into these incision sites to maintain its patency and promote the formation of collaterals. Endolaser treatment was then performed around the incision site followed by panretinal laser photocoagulation.

Of 16 attempted chorioretinal venous anastomosis sites, 10 were functioning. In all cases there was proliferation of avascular fibrous tissue. Final visual acuity was better in three eyes. Larger trials are awaited.

\section{INTERVENTIONS FOR BRVO \\ Steroids}

Similar to their use in CRVO, the use of intravitreal triamcinolone has recently been advocated in BRVO patients and, in a controlled study, was shown to significantly improve visual acuity in patients with chronic macular oedema. ${ }^{26}$ Further work is awaited. The risks of this treatment are comparable to those for CRVO.

\section{Isovolaemic haemodilution}

Chen et al demonstrated positive results for isovolaemic haemodilution given up to 3 months after BRVO in a randomised controlled study; 18 patients were treated for 6 weeks and compared to 16 control patients. After 1 year of follow up the visual acuity in the treated group improved compared to the controls $(p=0.03)$. Final visual acuities were 6/12 and 6/24 for treated and untreated patients, respectively. At the 3 month follow up argon laser treatment was given for macular oedema and vision less than 6/12, neovascularisation or capillary non-operfusion greater than 5 disc diameters. There was no significant difference between cases and controls requiring laser treatment. It therefore appears that isovolaemic haemodilution may be of benefit in both CRVO and BRVO. ${ }^{43}$

\section{Vitrectomy}

The vitreous is postulated to have a role in the pathogenesis of neovascularisation and macular oedema, which may complicate BRVO and its removal may help in the management of these sight threatening complications.

An intact vitreoretinal surface provides a scaffold for neovascularisation. ${ }^{67}{ }^{68}$ The vitreous allows angiogenic factors from the ischaemic retina to diffuse into it and traction through the vitreous fibres on the Muller cells of the retina predisposes to cystoid macular oedema. ${ }^{69}$ Animal studies on vitrectomised and non-vitrectomised eyes show that inducing a BRVO in non-vitrectomised eyes results in retinal hypoxia, whereas BRVO induced in vitrectomised eyes produced no change in retinal oxygen tension. ${ }^{70}$

It is postulated that vitrectomy allows access of oxygenated aqueous to the inner retina, thereby improving macular oedema, reducing ischaemia, and improving vision. Pars plana vitrectomy has been shown to reduce macular oedema and restore the normal foveal contour but there was no significant change in best corrected visual acuity.

Visual improvement occurs after vitrectomy for vitreous haemorrhage or retinal detachment complicating BRVO. ${ }^{71} 72$ This is an established treatment for these complications. No studies are available on vitrectomy being performed before the development of these complications.

\section{Arteriovenous crossing sheathotomy}

Venous compression in BRVO may be relieved by dividing the common adventitial sheath that surrounds retinal arterioles and venules at arteriovenous (A-V) crossing points.

An initial case report in a 54 year old woman with a 3 week history of BRVO showed visual improvement from 6/ $60(20 / 200)$ to $6 / 7.5(20 / 25)$ after 8 months' follow up. ${ }^{73}$ However, the re-perfusion status was not indicated. In a small uncontrolled prospective series of 15 eyes, Opremcak and Bruce reported results after A-V sheathotomy with BRVO and $6 / 21(20 / 70)$ or worse Snellen visual acuity. ${ }^{74}$ In all patients, intraoperative sheath decompression was achieved, resulting in clinical improvements in retinal haemorrhages and retinal perfusion. Postoperative visual acuity improved in 10 of 15 patients $(67 \%)$ with an average of four lines of vision gained. Nevertheless, vision did not improve in three patients despite surgical success and resolution of fundus changes. This was attributed by the authors, to a postoperative IOP rise. Two patients developed vascular bleeding as a result of an anatomical variation in vessel configuration at the A-V crossing point of the BRVO. The authors did not report visual recovery to correlate with initial visual acuity, duration of the BRVO, severity of intraretinal haemorrhage, oedema or ischaemia, or with final visual acuity.

In a controlled prospective study 43 patients with BRVO were treated with A-V decompression while 25 patients who refused surgery acted as controls. ${ }^{75}$ The mean VA improved from 0.35 to 0.16 on the logMAR scale; 26 patients $(60 \%)$ gained at least two lines of vision and 12 patients (28\%) gained at least four lines of vision. After 6 weeks' follow up, macular oedema and retinal haemorrhages had resolved. Patients with the most improvement also showed angiographic improvements in venous perfusion, whereas four patients with completely occluded vessels showed the worst functional results. Despite visual improvement, capillary 
reperfusion was only observed in four eyes. Overall, the functional outcome were significantly better in patients with $\mathrm{A}-\mathrm{V}$ decompression than controls and outcomes were more favourable in patients under 65 years of age. ${ }^{75} 76$ This may be explained by the advanced sclerotic changes of the blood vessels in older patients.

Retrospective studies have similarly shown benefits from A-V sheathotomy. ${ }^{78}$ Although the case series are small, visual improvements of $80 \%$ have been demonstrated in patients with preoperative vision of $6 / 60$ (20/200). These studies have follow ups ranging from 5-7 years. Cahill et al report a complete resolution of macular oedema and a significant increase in visual acuity in one third of patients after A-V sheathotomy. ${ }^{79}$ However, in the majority of cases a reduction of oedema was not associated with improved visual acuity.

The optimum timing of intervention is unclear. Some investigators have reported successful surgical intervention after as long as 6 months. $^{74}$ In other studies if the preoperatively duration of BRVO was 3 months, the results were worse than those cases where a shorter interval occurred between BRVO and surgery.

Reported complications are few but include cataract, nerve fibre layer defects, haemorrhage, retinal tear, postoperative gliosis, and retinal detachment.

Although preliminary results are encouraging, further questions remain. What is the time window in which decompression would give optimal results? Will A-V sheathotomy be more effective if performed before the thrombus becomes organised? The success of A-V sheathotomy may be partly attributed to the vitrectomy performed at the same time which resulted in preventing neovascularisation and persistent macular oedema. ${ }^{80}$ Further investigations are required to better define what degree of functional deterioration caused by BRVO mandates surgical intervention. It may be that a combination approach is needed-such as $\mathrm{A}-\mathrm{V}$ sheathotomy and injection of thrombolytic into the occluded vein, which resulted in thrombus release in $28 \%$ cases and significant correlation with early surgery and better final visual acuity. ${ }^{81}$

\section{CONCLUSION}

Retinal vein occlusions are the second most common sight threatening vascular disorder. Despite this, our therapeutic armamentarium for functional improvement has been very limited. We are now moving into a new era where many novel interventional treatments have been described for RVOs.

There are three main deficiencies in the evidence we currently have for interventional treatments in RVO. The main observation is the lack of large well designed prospective randomised controlled trials. Pilot studies on interventional treatments for vein occlusion seem promising but all are non-randomised and uncontrolled studies in small numbers of patients. Randomised controlled trials remain the gold standard of research methodology and provide the best indication of treatment effect. Until these are available many ophthalmologists will be reluctant to change their practice.

Secondly, there is lack of long term follow up in many studies. While some interventions (for example, intravitreal steroids) seem to have a temporary effect, we do not know the long term outcomes of other measures. The third issue to address is optimal timing of intervention and patient selection. Ideally, early intervention would be best before thrombus organisation or further ischaemic damage occurs but the effective treatment window remains obscure. Most interventions appear to be more successful in non-ischaemic vein occlusions but further clarification is necessary.
At present the evidence to date does not support any therapeutic benefit from radial optic neurotomy, optic nerve sheath decompression or $\mathrm{A}-\mathrm{V}$ crossing sheathotomy in eyes with vein occlusion. Furthermore, the potential complications outweigh any benefits. The use of isovolaemic haemodilution requires careful patient selection and should be avoided in patients with concurrent cardiovascular, renal, or pulmonary disease. Unfortunately, as a significant number of patients with vein occlusion have concurrent cardiovascular morbidity, and the benefits are limited, we would not recommend haemodilution.

With the available evidence we have we suggest that the use of intravitreal steroids may be a beneficial early intervention in carefully selected patients with CRVO or BRVO. Intravitreal steroids have been evaluated in nonrandomised comparative studies and have been shown to be significantly better than no treatment, and although the steroid effect is not permanent it may prevent early photoreceptor damage from macular oedema and improve long term prognosis. Anti-VEGF agents, which are gaining popularity in treatment of choroidal neovascularisation, may have a role in future.

Vitrectomy with intravenous thrombolysis with t-PA seems to offer the most promising option for CRVO. This procedure seems to give the best outcome with fewer side effects. It also seems the most physiological of all the presently suggested options. Similarly, although not tried yet, vitrectomy, A-V sheathotomy combined with intravenous t-PA may offer benefits in BRVO.

Despite uncertainty and open questions, surgical interventions are likely to be a therapeutic option for RVO in the future. Vein occlusions occur at a sufficiently high frequency for future prospective randomised controlled studies to be conducted so that we can truly evaluate the role of each therapeutic modality both individually and in combination.

\section{Authors' affiliations}

H Shahid, Prince Charles Eye Unit, King Edward VII Hospital, Windsor SL4 3DP, UK

P Hossain, Department of Ophthalmology, Southampton General Hospital, Southampton SO16 6YD, UK

W M Amoaku, Academic Department of Ophthalmology, University of Nottingham, Eye, Ear, Nose and Throat Centre, Queen's Medical

Centre, Nottingham NG7 2UH, UK

Proprietary interest: none.

Competing interests: none declared

\section{REFERENCES}

1 Branch Vein Occlusion Study Group. Argon laser photocoagulation for macular edema in branch vein occlusion. Am J Ophthalmol 1984;98:271-82.

2 Hayreh SS, Zimmerman MB, Podhajsky P. Incidence of various types of retinal vein occlusion and their recurrence and demographic characteristics. Am J Ophthalmol 1994; 117:429-41.

3 Central Vein Occlusion Study Group. Natural history and clinical management of central retinal vein occlusion. Arch Ophthalmol 1997;115:486-91.

4 Central Vein Occlusion Study Group. Baseline and early natural history report. Arch Ophthalmol 1993;111:1087-95.

5 Mitchell P, Smith W, Chang A. Prevalence and associations of retinal vein occlusion in Australia. The Blue Mountains Eye Study. Arch Ophthalmol 1996;114:1243-7.

6 Quinlan PM, Elman MJ, Bhatt AK, et al. The natural course of central retinal vein occlusion. Am J Ophthalmol 1990;110:118-23.

7 Zegarra H, Gutman FA, Conforto J. The natural course of central retinal vein occlusion. Ophthalmology 1979;86:1931-42.

8 Central Vein Occlusion Study Group M Report. Evaluation of grid pattern photocoagulation for macular oedema in central vein occlusion. Ophthalmology 1995;102:1425-33.

9 Branch Vein Occlusion Study Group. Argon laser scatter photocoagulation for prevention of neovascularization and vitreous hemorrhage in branch vein occlusion. A randomized clinical trial. Arch Ophthalmol 1986;104:34-41.

10 Hayreh SS. Central retinal vein occlusion: differential diagnosis and management. Trans Am Acad Ophthalmol Otolaryngol 1977;83(3 Pt 1):OP379-91. 
11 Shaikh S, Blumenkranz MS. Transient improvement in visual acuity and macular edema in central retinal vein occlusion accompanied by inflammatory features after pulse steroid and anti-inflammatory therapy. Retina $2001 ; 21: 176-8$

12 Degenring RF, Kamppeter B, Kreissig I, et al. Morphological and functional changes after intravitreal triamcinolone acetonide for retinal vein occlusion. Acta Ophthalmol Scand 2003;81:548-50.

13 Greenberg PB, Martidis A, Rogers AH, et al. Intravitreal triamcinolone acetonide for macular oedema due to central retinal vein occlusion. Br J Ophthalmol 2002;86:247-8.

14 Ip M, Kahana A, Altaweel M. Treatment of central retinal vein occlusion with triamcinolone acetonide: an optical coherence tomography study. Sem Ophthalmol 2003;18:67-73.

15 Ip MS, Kumar KS. Intravitreous triamcinolone acetonide as treatment for macular edema from central retinal vein occlusion. Arch Ophthalmol 2002;120:1217-19

16 Jonas JB, Kreissig I, Degenring RF. Intravitreal triamcinolone acetonide as treatment of macular edema in central retinal vein occlusion. Graefes Arch Clin Exp Ophthalmol 2002;240:782-3.

17 Park CH, Jaffe GJ, Fekrat S. Intravitreal triamcinolone acetonide in eyes with cystoid macular edema associated with central retinal vein occlusion. Am J Ophthalmol 2003;136:419-25.

18 Ip MS, Gottlieb JL, Kahana A, et al. Intravitreal triamcinolone for the treatment of macular oedema associated with central retinal vein occlusion. Arch Ophthalmol 2004;122:1131-6.

19 Bashshur ZF, Ma'luf RN, Allam S, et al. Intravitreal triamcinolone for the management of macular oedema due to nonischaemic central retinal vein occlusion. Arch Ophthalmol 2004;122:1137-40.

20 Antcliff RJ, Spalton DJ, Stanford MR, et al. Intravitreal triamcinolone for uveitic cystoid macular oedema: an optical coherence tomography study. Ophthalmology 2001;108:765-72.

21 Wilson CA, Berkowitz BA, Sato Y, et al. Treatment with intravitreal steroid reduces blood-retina barrier breakdown due to retinal photocoagulation. Arch Ophthalmol 1992;110:1155-9.

22 Nauck M, Karakiulakis G, Perrochoud AP, et al. Corticosteroids inhibit the expression at the vascular endothelial growth factor gene in human vascular smooth muscle cells. Eur J Pharmacol 1998:341:309-15.

23 Jonas JB, Hayler JK, Sofker A, et al. Regression of neovascular iris vessels by intravitreal injection of crystalline cortisone. J Glaucoma $2001 ; 10: 284-7$.

24 Chen SD, Lochhead J, Patel CK, et al. Intravitreal triamcinolone acetonide for ischaemic macular oedema caused by branch retinal vein occlusion. Br J Ophthalmol 2004;88:154-5.

25 Jonas JB, Akkoyun I, Kamppeter B, et al. Branch retinal vein occlusion treated by intravitreal triamcinolone acetonide. Eye, April 2004; Advance e-pub.

26 Kaushik S, Gupta V, Gupta A, et al. Intractable glaucoma following intravitreal triamcinolone in central retinal vein occlusion. Am J Ophthalmol 2004; 137:758-60

27 Duff IF, Falls HF, Linman JW. Anticoagulant therapy in occlusive vascular disease of the retina. Am Arch Ophthalmol 1951;46:601-17.

28 Vannas S, Ritta C. On anticoagulant treatment of occlusion of the central vein of the retina. Acta Ophthalmol 1968;46:730-6.

29 Kohner EM, Pettit JE, Hamilton AM, et al. Streptokinase in central retinal vein occlusion: a controlled clinical trial. BMJ 1976;1:550-3.

30 Elman MJ. Thrombolytic therapy for central retinal vein occlusion: results of a pilot study. Trans Am Ophthal Soc 1996;XCIV:471-504.

31 Lahey JM, Fong DS, Kearney J. Intravitreal tissue plasminogen activator for acute central retinal vein occlusion. Ophthalmic Surg Lasers 1999;30:427-34

32 Glacet-Bernard A, Kuhn D, Vine AK, et al. Treatment of recent onset central retinal vein occlusion with intravitreal tissue plasminogen activator: a pilot study. Br J Ophthalmol 2000;84:609-13.

33 Loren L, Frade G, Torrado MC, et al. Thrombus age and tissue plasminogen activator mediated thrombolysis in rats. Thromb Res 1989:56:67-76.

34 Elman MJ, Raden RZ, Carrigan A. Intravitreal injection of tissue plasminogen activator for central retinal vein occlusion. Trans Am Ophthal Soc $2001 ; 99: 219-23$

35 Ghazi NG, Noureddine B, Haddad RS, et al. Intravitreal tissue plasminogen activator in the management of central retinal vein occlusion. Retina 2003;23:780-4.

36 Weiss JN. Treatment of central retinal vein occlusion by injection of tissue plasminogen activator into a retinal vein. Am J Ophthalmol 1998; 126: 142-4

37 Weiss JN, Bynoe LA. Injection of tissue plasminogen activator into a branch retinal vein in eyes with central retinal vein occlusion. Ophthalmology 2001;108:2249-57.

38 Hayreh SS. t-PA in CRVO. Ophthalmology 2002;109:1758-61.

39 Paques M, Vallee JN, Herbreteau D, et al. Superselective ophthalmic artery fibrinolytic therapy for the treatment of central retinal vein occlusion. Br J Ophthalmol 2000;84:1387-91.

40 Vallee JN, Massin P, Aymard A, et al. Superselective ophthalmic arterial fibrinolysis with urokinase for recent severe central retinal venous occlusion: initial experience. Radiology 2000;216:47-53.

41 Vallee JN, Pacques M, Aymard A, et al. Combined central retinal arterial and venous obstruction: emergency ophthalmic arterial fibrinolysis. Radiology 2002;223:351-9.

42 Jorizzo PA, Klein ML, Shults WT, et al. Visual recovery in combined central artery and central vein occlusion. Am J Ophthalmol 1987;104:358-63.
43 Chen HC, Wiek J, Gupta A, et al. Effect of isovolaemic haemodilution on visual outcome in branch retinal vein occlusion. $\mathrm{Br} J$ Ophthalmol 1998;82:162-7.

44 Glacet-Bernard A, Zourdani A, Milhoub M, et al. Effect of isovoemic hemodilution in central retinal vein occlusion. Graefes Arch Clin Exp Ophthalmol 2001;239:909-14.

45 Hansen LL, Danisevskis P, Arntz H-R, et al. A randomised prospective study on treatment of central retinal vein occlusion by isovolaemic haemodilution and photocoagulation. Br J Ophthalmol 1985;69:108-16.

46 Hansen LL, Wiek J, Wiederholt $M$, et al. A randomised prospective study of treatment of non-ischaemic central retinal vein occlusion by isovolaemic haemodilution. Br J Ophthalmol 1989;73:895-9.

47 Opremcak EM, Bruce RA, Lomeo MD, et al. Radial optic neurotomy for central retinal vein occlusion: a retrospective pilot study of 11 consecutive cases. Retina $2001 ; 21: 408-15$.

48 Bynoe LA, Opremcak EM, Bruce RA, et al. Radial optic neurotomy for central retinal vein obstruction. Retina 2002;22:379-80.

49 Hayreh SS, Opremcak EM. Radial optic neurotomy for central retinal vein occlusion. Retina 2002;22:374-7.

50 Weizer JS, Stinnett SS, Fekrat S. Radial optic neurotomy as treatment for central retinal vein occlusion. Am J Ophthalmol 2003;136:814-19.

51 Garciia-Arumii J, Boixadera A, Martinez-Castillo V, et al. Chorioretinal anastomosis after radial optic neurotomy for central retinal vein occlusion. Arch Ophthalmol 2003;121:1385-91.

52 Williamson TH, Poon W, Whitefield N, et al. A pilot study of pars plana vitrectomy, intraocular gas, and radial neurotomy in ischaemic central retinal vein occlusion. Br J Ophthalmol 2003;87:1 126-9.

53 Guajardo LL, de la Huerga AM, Sandomingo AF, et al. Radial optic neurotomy as a treatment of central vein occlusion: neurotomy in central vein occlusion. Retina 2003;23:890.

54 Hayreh SS. Radial optic neurotomy for nonischemic central retinal vein occlusion. Arch Ophthalmol 2004;122:1572-3.

55 Vasco-Pasada J. Modification of the circulation in the posterior pole of the eye. Am J Ophthalmol 1972;1:48-59.

56 Arcinegas A. Treatment of the occlusion of the retinal vein by section of the posterior ring. Ann Ophthalmol 1984;16:1081-6.

57 Lee SY, Shin OH, Spoor TO, et al. Bilateral retinal venous calibre decrease following unilateral optic nerve sheath decompression. Ophthalmic Surg 1995;26:25-8

58 Dev S, Buckley EG. Optic nerve sheath decompression for progressive central retinal vein occlusion. Ophthalmic Surg Lasers 1999;30:181-4.

59 Lit ES, Tsilimbaris M, Gotzaridis E, et al. Lamina puncture: pars plana optic disc surgery for central retinal vein occlusion. Arch Ophthalmol 2002;120:495-9.

60 Verhoeff FH. Successful diathermy treatment in a case of recurring retinal haemorrhages and retinitis proliferans. Arch Ophthalmol 1948;40:239-44.

61 Kwok AKH, Lee VYW, Hon C. Laser induced chorioretinal venous anastomosis in ischaemic central retinal vein occlusion. Br J Ophthalmol 2003;87:1043-55

62 McAllister IL, Douglas JP, Constable IJ, et al. Laser-induced chorioretinal venous anastomosis for nonischaemic central retinal vein occlusion: evaluation of the complications and their risk factors. Am J Ophthalmol 1998; 126:219-29.

63 McAllister IL, Constable IJ. Laser-induced chorioretinal venous anastomosis for treatment of nonischaemic central retinal vein occlusion. Arch Ophthalmol $1995 ; 113: 456-62$

64 Fekrat S, Goldberg MF, Finkelstein D. Laser-induced chorioretinal venous anastomosis for nonischaemic central or branch retinal vein occlusion. Arch Ophthalmol 1998;116:43-52

65 Parodi MB. Laser-induced chorioretinal anastomosis and central retinal vein occlusion. Arch Ophthalmol 1999;117:140-1.

66 Peyman GA, Kishore K, Conway MD. Surgical chorioretinal venous anastomosis for ischaemic central retinal vein occlusion. Ophthalmic Surg Lasers 1999:30:605-14.

67 Avunduk AM, Cetinkaya K, Kapicioglu Z, et al. The effect of posterior vitreous detachment on the prognosis of branch retinal vein occlusion. Acta Ophthalmol Scand 1997;75:441-2.

68 Takahashi MK, Hikichi T, Akiba J, et al. Role of the vitreous and macular oedema in branch retinal vein occlusion. Ophthalmic Surg Lasers 1997; 28:294-9.

69 Sebag J, Balazs EA. Pathogenesis of cystoid macular oedema: an anatomic consideration of vitreoretinal adhesions. Surv Ophthalmo 1984;28(suppl):493-8.

70 Stefansson E, Novack RL, Hatchell DL. Vitrectomy prevents retinal hypoxia in branch retinal vein occlusion. Invest Ophthalmol Vis Sci 1990;31:284-9.

71 Yeshaya A, Treister G. Pars plana vitrectomy for vitreous haemorrhage and retinal vein occlusion. Ann Ophthalmol 1983;15:615-17.

72 Amirikia A, Scott IU, Murray TG, et al. Outcomes for vitreoretinal surgery for complications of branch retinal vein occlusion. Ophthalmology $2001 ; 108: 372-6$.

73 Osterloh MD, Charles S. Surgical decompression of branch retinal vein occlusions. Arch Ophthalmol 1988;106:1469-71.

74 Opremcak EM, Bruce RA. Surgical decompression of branch retinal vein occlusion via arteriovenous crossing sheathotomy: a prospective review of 15 cases. Retina 1999;19:1-5.

75 Mester U, Dillinger P. Vitrectomy with arteriovenous decompression and internal limiting membrane dissection in branch retinal vein occlusion. Retina $2002 ; 22: 740-6$ 
76 Charbonnel J, Glacet-Bernard A, Korobelnik J, et al. Management of branch retinal vein occlusion with vitrectomy and arteriovenous adventitial sheathotomy, the possible role of surgical posterior vitreous detachment. Graefes Arch Clin Exp Ophthalmol 2004;242:223-8.

77 Shah GK, Sharma S, Fineman MS. Arteriovenous adventitial sheathotomy for the treatment of macular oedema associated with branch retinal vein occlusion. Am J Ophthalmol 2000;129:104-6.

78 Shah GK. Adventitial sheathotomy for treatment of macular oedema associated with branch retinal vein occlusion. Curr Opin Ophthalmol 2000;11:171-4
79 Cahill MT, Kaiser PK, Sears JE, et al. The effect of arteriovenous sheathotomy on cystoid macular oedema secondary to branch retinal vein occlusion. $\mathrm{Br}$ J Ophthalmol 2003;87:1329-32.

80 Le Rouic JF, Beijani RA, Rumen F, et al. Adventitial sheathotomy for decompression of recent onset branch retinal vein occlusion. Graefes Arch Clin Exp Ophthalmol 2001;239:747-51.

81 Garcia-Arumi J, Martinez-Castillo V, Boixadera A, et al. Management of macular oedema in branch retinal vein occlusion with sheathotomy and recombinant tissue plasminogen activator. Retina 2004;24:530-40. 